\title{
Parents and Teachers Make Different Contributions to a Shared Perspective on Hyperactive-Impulsive and Inattentive Symptoms: A Multivariate Analysis of Parent and Teacher Ratings on the Symptom Domains of ADHD
}

\author{
Gráinne McLoughlin • F. Rijsdijk • P. Asherson • \\ J. Kuntsi
}

Received: 13 July 2010/Accepted: 4 May 2011/Published online: 10 June 2011

(C) Springer Science+Business Media, LLC 2011

\begin{abstract}
Attention deficit hyperactivity disorder (ADHD) is characterised by developmentally inappropriate and impairing levels of inattentive and hyperactive-impulsive behaviours. We aimed to investigate the differential effects of parent and teacher ratings on inattention and hyperactivity-impulsivity and the extent of genetic overlap between the two behavioural dimensions. Multivariate structural equation modelling was performed on DSM-IV based ADHD ratings by parents and teachers collected on a general population sample of 672 twin pairs, at ages 7-10 years. This study is the first to simultaneously use parent and teacher ratings in twin modelling to examine the effects of different raters on the two behavioural dimensions of ADHD. The findings indicated that hyperactivity-impulsivity and inattention load on to separate latent factors that represent a common behavioural view for both parents and teachers, although there are additional aspects to the observations of these behaviours that are unique to each type of rater. The findings further indicate some shared aetiology for hyperactivity-impulsivity and inattention as measured by both parent and teacher ratings, in agreement with previous findings on the aetiology of the two symptom dimensions of ADHD.
\end{abstract}

Keywords Twin study - Attention deficit hyperactivity disorder · Hyperactivity-impulsivity - Inattention · Parent ratings $\cdot$ Teacher ratings

Edited by Michael Lyons.

G. McLoughlin ( $\square)$ · F. Rijsdijk · P. Asherson · J. Kuntsi MRC Social, Genetic and Developmental Psychiatry Centre, Institute of Psychiatry, King's College London, De Crespigny Park, London SE5 8AF, UK

e-mail: grainne.mcloughlin@kcl.ac.uk

\section{Introduction}

The current edition of the Diagnostic and Statistical Manual of Mental Disorders, (DSM-IV; APA 2000) divides the behavioural symptoms of ADHD into two separate symptom groups, one reflecting inattention and the other reflecting a combination of hyperactivity and impulsivity (Burns et al. 1997; Conners et al. 1998b; Conners 1998; Hudziak et al. 1998), as suggested by factor analyses (e.g. Pelham et al. 1992; McLoughlin et al. 2007). The two symptom dimensions are expressed at varying levels in children, leading to individual differences in the balance of symptoms, reflected in the DSM-IV ADHD diagnoses of primarily inattentive, primarily hyperactive-impulsive and combined type ADHD.

The dyadic classification of ADHD symptomatology has stimulated research on the extent to which the two dimensions share the same aetiological pathways. One approach that has been used to delineate the aetiological relationship between hyperactivity-impulsivity and inattention is the twin study design. Thus far, twin studies have focused on the extent to which hyperactivity-impulsivity and inattention have shared or unique genetic and environmental influences. A small study of 188 male twin pairs aged 11 and 12 years using parent and teacher ratings of DSM-III symptoms, indicated similar genetic correlations ( $\mathrm{rG}$ : indicating the degree of shared genetic influences between two measures) between the two symptom dimensions for teacher $(\mathrm{rG}=0.58)$ and parent ratings $(\mathrm{rG}=0.60)$ (Sherman et al. 1997). A second larger twin study of 1,376 twin pairs, using parent reported interview data with the Child and Adolescent Psychiatric Assessment, found high genetic correlations (mother: $\mathrm{rG}=0.50-0.97$; father $\mathrm{rG}=(0.58-0.89)$ among the three DSM-IIIR defined behaviours of inattention, hyperactivity and impulsivity in $8-16$ year olds (Eaves et al. 
2000). Another study examined genetic influences contributing to the development of hyperactive-impulsive and inattentive symptoms at three developmental stages from childhood to adolescence (8-9, 13-14, and 16-17 years) in a sample of 824 twins using DSM-III- and DSM-IV-based parent ratings with similar results at each developmental stage (Larsson et al. 2006). The most recent study using parent ratings of DSM-IV symptoms in a larger sample of 6,222 pairs, aged approximately 8 years, again found comparable results with substantial genetic overlap $(\mathrm{rG}=0.57$ for boys; $\mathrm{rG}=0.62$ for girls) between hyperactivityimpulsivity and inattention (McLoughlin et al. 2007). Overall the previous twin studies are consistent in finding substantial genetic overlap between behavioural ratings of inattention and hyperactivity-impulsivity, but importantly, they also identify specific genetic influences that act separately on each of the two symptom domains.

The various twin studies to date that have investigated the aetiological relationship between hyperactivity-impulsivity and inattention therefore indicate that there are both common and unique genetic effects between the two symptoms domains. One question that is not well addressed in the previous literature, however, is the degree to which the choice of rater affects the estimation of shared and unique aetiological influences on rating scale measures of inattention and hyperactivity-impulsivity. This is an important question since both parent and teacher ratings are typically used when evaluating children with ADHD, and persistence of symptoms across different situations (e.g. home and school) are an important part of clinical diagnostic criteria. Previous studies have found that parents and teachers show only moderate agreement in their ratings of the full ADHD phenotype, with a modest correlation of around 0.3 (reviewed in Kuntsi and Asherson 2005). This low correlation has been attributed to differences in the way parents and teachers perceive and rate child behaviour, as well as differences in child behaviour in home and school settings (Saudino 2005). Disagreement between parents and teachers in ratings of ADHD behaviour could have consequences for genetic studies, as the results of these studies partially depend on how one defines and measures ADHD symptoms. If parents and teachers differ in ratings of ADHD behaviour, the phenotype being measured may also differ depending on who rates the child, therefore influencing associations with genetic or environmental measures. This is perhaps indicated by differences in heritability estimates of parent and teacher ratings with parent ratings often giving rise to slightly higher heritabilities than teacher ratings (Eaves et al. 1997; Goodman and Stevenson 1989); although this is not always the case (Martin et al. 2002; Saudino et al. 2005) and could be due the use of different teachers rating members of a twin pair, whereas it is usually the mother who rates both children. However, partially distinct aetiologies for the parent ratings of ADHD and teacher ratings of ADHD are indicated by rater-specific genetic effects, in addition to shared genetic effects (Martin et al. 2002; Nadder et al. 2001; Thapar et al. 2000). These shared and specific rater effects suggest that, for molecular genetic studies on the full ADHD phenotype, there is value in mapping genetic variants that are associated with both parent and teacher ratings, as well as those that are specific to each rater (Holmes et al. 2002). The presence of unique genetic factors has further implications for understanding the underlying neurological processes involved, since these imply that domain specific neurobiological networks are likely to exist. Common genetic effects could in addition indicate shared neurobiological processes, but could also reflect the multiple distinct outcomes of genes, involving two or more pathways.

With the exception of one small study (Sherman et al. 1997), previous investigations of the phenotypic and etiological relationship between hyperactivity-impulsivity and inattention have focused on parent ratings and none have examined these behaviours using models that take into account multiple raters. As a result, the extent to which parents and teachers generate comparable measures of the two symptom domains of ADHD and the extent to which the simultaneous examination of parent and teacher ratings change the estimation of the genetic overlap between hyperactivity-impulsivity and inattention, remains unknown. We therefore set out in this study to compare models that make different assumptions about the effects of parent and teacher ratings on the phenotypic and etiological overlap between hyperactivity-impulsivity and inattention.

We first take a non-restrictive view that assumes that parents and teachers are rating different aspects of the two behavioural dimensions of ADHD. For example, this could arise if parents and teachers report behaviours observed in distinct situations or if they do not share a common understanding of the behaviours. The view of parent and teachers rating different aspects of behaviour is represented by a standard multivariate genetic and environmental correlated factors model, also known as the biometric model (Hewitt et al. 1992), where the variables are the parent and teacher ratings of hyperactivity-impulsivity and inattention.

We then examine a second model that assumes that parent and teacher ratings converge in a common behavioural view that encompasses both hyperactivity-impulsivity and inattention, but with the rater specific scores also reflecting additional aspects of behaviour that are unique to each raters perspective of the child's behaviour. This model is known as the psychometric or common pathway model (Hewitt et al. 1992).

Our final model extends the common pathway model by separating hyperactivity-impulsivity and inattention into 
two distinct factors that reflect the shared view of parents and teachers but, similar to the previous model, with rater specific scores reflecting additional rater specific views of the child's behaviour.

Behavioural perspectives that are unique to each rater may occur when the rater observes the child in distinct situations, but may also reflect differences in understanding and interpretation of child behaviour by parents and teachers. For example, teachers observe children in the school environment, which may elicit behaviours that parents do not observe at home, and parents and teachers may have different expectations of what is appropriate in different situations. As such, the unique behavioural view provides different contributions from the perspective of each rater (van der Valk et al. 2001); whereas, the common behavioural view is stable across situation and independent of rater specific effects.

Evidence for a shared environmental contribution to the unique rater view may indicate rater bias effects because shared environmental influences that are specific to teacher or parent ratings can represent systematic response styles where parents or teachers are biasing the ratings of both twins in the same way (Saudino 2005). As neither rater bias nor unreliability can result in the systematic effects necessary for the model to estimate genetic influences, the specific genetic effects represent real effects that are unique to each rater (van der Valk et al. 2001; Bartels et al. 2004; Saudino 2005).

In order to better understand the influence of different rater perspective on our understanding of the aetiological influences on the two symptom domains of ADHD, we applied these three models to hyperactive-impulsive and inattention ratings from a sample of 672 twin pairs aged 7-10 years. We sought to examine whether parents and teachers have a shared rater view and rate the same hyperactive-impulsive and inattentive phenotypes. We further examine the effects of different raters on the estimate of the genetic overlap between hyperactivity-impulsivity and inattention.

\section{Method}

Sample

Participants are members of the Study of Activity and Impulsivity Levels in children (SAIL) (Kuntsi et al. 2006), a study of a general population sample of twins aged 7-10 years. The sample was recruited from a birth cohort study, the Twins Early Development Study (TEDS; Trouton et al. 2002), which had invited parents of all twins born in England and Wales during 1994-1996 to enroll. Despite attrition, the TEDS families continue to be reasonably representative of the UK population with respect to parental occupation, education and ethnicity (Oliver and Plomin 2006). Zygosity was determined using a standard zygosity questionnaire that has been shown to have 95\% accuracy (Price et al. 2000). Families on the TEDS register were invited to take part if they fulfilled the following SAIL project inclusion criteria: twins' birthdates between September 1, 1995, and December 31, 1996; lived within a feasible travelling distance from the research centre; ethnic origin White European (to reduce population heterogeneity for molecular genetic studies); recent participation in TEDS, as indicated by return of questionnaires at either 4- or 7-year data collection point; no extreme pregnancy or perinatal difficulties or specific medical syndromes, chromosomal anomalies, or epilepsy; not participating in other current TEDS sub-studies. Further, as this study also included cognitive testing, none of the children in the sample was on stimulant or other neuropsychiatric medications. For cognitive testing, it is important to investigate these processes in children who are not on medication. Of the 1,230 suitable families contacted, 672 families agreed to participate, reflecting a participation rate of $55 \%$. Thirty individual children were subsequently excluded because of intelligence quotient (IQ) $<70$, epilepsy, autism, obsessive-compulsive disorder or neurodevelopmental disorder, illness during testing, or placement on stimulant medication for ADHD. Therefore, the final sample consisted of 1,314 individuals (651 male and 663 female), with full data from both twins in a pair for 646 families and single twin data for 22 families. Monozygotic (MZ) twins were $39 \%$ of the sample $(n=514), 28.5 \%$ of the sample belonged to same-sex dizygotic (DZ) pairs and $32.5 \%$ of the sample were opposite-sex dizygotic pairs (DZOS). The mean age for the sample was 8.83 years ( $\mathrm{SD}=0.67$; range 7.92-10.92 years). Participating families gave their informed consent and the study was approved by the Institute of Psychiatry Ethical Committee.

\section{Measures}

Parents and teachers completed the Long Version of the Conners' Parent Rating Scale (Conners et al. 1998b) and the Long Version of the Conners' Teacher Rating Scale (Conners et al. 1998a), which included the hyperactiveimpulsive and inattentive DSM-IV ADHD symptom subscales. The primary caregiver completed parent ratings, which in most cases was the mother. Teacher data were completed by the main class teacher for each child. The rater indicated on a four-point scale how well each attribute described the child: not true at all (0), just a little true (1), pretty much true (2), very much true (3). Items include, for example, "is always on the go or acts as if driven by a motor" and "has difficulty sustaining attention in tasks or 
play activities." In a small number of cases (PC hyperactive-impulsive: 11 cases; PC inattentive: 9 cases; TC hyperactive-impulsive: 22 cases; TC inattentive: 13 cases), missing data in Conners' scales were pro-rated, where a summary score based on the mean of individual questions on the rest of the subscale was used, if there was more than $75 \%$ completion for each subscale.

\section{Quantitative genetic analyses}

The structural equation model-fitting program Mx (Neale 1997) was used to conduct the genetic analyses. Models were fitted to age- and sex-regressed residual scores, using raw data analysis, rather than covariance matrices. The advantage of this approach is that participants with incomplete data can be included in the analyses, as $\mathrm{Mx}$ provides a method for handling incomplete data by using raw maximum likelihood estimation, in which a likelihood statistic (-2LL) of the data for each observation is calculated. The use of raw maximum likelihood estimation implies that there is no overall measure of fit (such as a $\chi^{2}$ value with corresponding $p$-value for the number of degrees of freedom, as obtained by fitting directly on observed variance-covariance matrices). Instead, with raw data, there are relative measures of fit: by comparing the $-2 \mathrm{LL}$ (and degrees of freedom) of our models with the $-2 \mathrm{LL}$ (and degrees of freedom) of the full saturated phenotypic model where the maximum number of free parameters is estimated to describe the correlational structure between variables. The saturated model provides a baseline comparison for subsequent genetic models. The comparison of the saturated model with subsequent genetic models provides a likelihood ratio chi square test of goodness-off fit.

The difference between the measure of fit of the saturated model and the genetic model is distributed as a chisquare $\left(\chi^{2}\right)$ with degrees of freedom (df) equivalent to the difference in the number of parameters between the models (Neale and Cardon 1992). A $\chi^{2}$-difference test can be performed to compare the fit of nested models. For nonnested models, the Akaike's information criterion (AIC) was used to determine the best-fitting and most parsimonious model. AIC was computed as $\chi^{2}$ minus $2 \mathrm{df}$ where the $\chi^{2}$ is the difference in -2 LL between the saturated and restricted model and df denotes the difference in degrees of freedom between the two models. The model with the lowest AIC value is considered to be the most parsimonious by this criterion (Akaike 1987).

Information about the precision of parameter estimates and their explained variance in $\mathrm{Mx}$ was obtained by likelihood-based confidence intervals (CIs) rather than by standard errors. In this method a parameter is progressively moved away from its maximum likelihood estimate in either direction (while the other model parameters are optimised) until the difference in fit, distributed as a chisquare with one degree of freedom, is significant. For $95 \%$ CI the 0.05 level of significance is approximately 3.84 chisquare units in each direction (Neale and Miller 1997).One of the assumptions of twin modelling is that the data are normally distributed; because these data were negatively skewed, a log transformation was used. A test of normality was conducted (sktest; Stata Corporation 1997), which simultaneously tests for skewness and kurtosis. It indicated that the transformed hyperactivity-impulsivity and inattention scores met the normality assumption $(p>0.05)$.

\section{Multivariate models}

Multivariate models begin with the cross-trait phenotypic correlation, which indicates the degree to which two variables covary. Phenotypic covariance between two variables can be decomposed into shared genetic and environmental influences using multivariate genetic analysis (Plomin et al. 2001): additive genetic (A), shared environment (C), which refers to experiences that make children growing up in the same family similar), and non-shared (child-specific) environment $(E)$, which refers to environmental influences that do not contribute to the similarity of children growing up in the same family and also includes measurement error. The parameter estimates are based on the assumption that there are no effects of assortative mating or gene-environment interaction or correlation. The bases of multivariate twin analysis are the cross-trait cross-twin correlations (i.e., the correlation between one twin's inattention and their co-twin's hyperactive-impulsive score). Multivariate twin analyses compare MZ and DZ cross-trait cross-twin correlations to estimate the extent to which phenotypic correlation is due to genes or environment. The genetic contribution to the phenotypic correlation is called bivariate heritability. This is the extent to which shared genetic influence generates a correlation between the two traits. From bivariate heritability, a statistic, called the genetic correlation, can be derived. The genetic correlation indicates the genetic overlap between two traits and varies from 0 (no genetic overlap) to 1 (complete genetic overlap between two traits). Therefore, the genetic correlation indicates the extent to which the same genetic factors are contributing to the two traits (Neale and Maes 1996). Importantly, the genetic correlation is independent of the heritability of the two traits; that is, the heritability of both traits could be high but the genetic correlation between them could be low, and vice versa. Similarly, in the bivariate model there are also shared and child-specific environmental correlations ( $\mathrm{rC}$ and $\mathrm{rE}$ ), which represent overlap in environmental influences between two phenotypes, and can also vary between 0 and 1 . To maximize 
power, in the multivariate analyses, no sex differences in variance components were modelled. Three standard multiple rater models were then employed, the correlated factors or biometric model and two common pathway or psychometric models, with one and two latent factors respectively (Hewitt et al. 1992).

\section{Correlated factors/biometric model (Fig. 1a)}

The extent to which the influences underlying parent and teacher ratings are shared is estimated in this model. So as to not give precedence to the latent variables underlying the first factor (here: parents' ratings), a correlated factor solution of the Cholesky model is presented. The correlated factors model is mathematically equivalent to the triangular decomposition, where the variance in each rating is decomposed into $\mathrm{A}, \mathrm{C}$ and $\mathrm{E}$ influences, and the correlations between variance components for each rater are estimated. The correlated factors model is also known as the biometric model as it provides a comparison for the subsequent common pathway or psychometric models, as it makes no assumptions regarding covariation between ratings of the same behaviour (Hewitt et al. 1992). The implication is that parents and teachers report on behaviours that are distinct from each other but may be correlated. This distinction may arise because the parents observe situationally-specific behaviours or have different understandings of the behavioural descriptions.

Common pathway/psychometric model: one latent factor (Fig. 1b)

In this model, it is suggested that correlations between raters arise because they are assessing a common phenotype that is influenced by genetic and/or environmental influences. The phenotypic variance for each twin is decomposed into $\mathrm{A}, \mathrm{C}$, and $\mathrm{E}$ that is common to both raters and rating subscales, as well as $\mathrm{A}, \mathrm{C}$, and $\mathrm{E}$ that is unique to each rater and rating subscales (Fig. 1b). Thus, according to this model, behavioural ratings include a phenotype common to both raters and specific phenotypes unique to each rater. In this model, the common phenotype represents reliable trait variance but the shared environmental component unique to each rater (significant rater-specific C) may be confounded by rater bias effects when the twin pairs are assessed by the same rater (Saudino 2005). Shared environmental influences that are specific to teacher or parent ratings can represent systematic response styles where parents or teachers are biasing the ratings of both twins in the same way thus increasing the $\mathrm{C}$ component and, as it's rater-specific, in a way that differs from the other rater (Hartman et al. 2007). Common A, C, and E are estimated from the cross twin correlations, and the unique rater/rating view is estimated from the difference between the variance shared between raters and the total variance.

Common pathway/psychometric model: two latent factors (Fig. 1c)

This model extends the common pathway model with one latent factor so that there are two latent factors explaining variance in each subscale-one for hyperactivity-impulsivity and one for inattention. The variance is again decomposed into that which is shared and that which is unique.

We first fitted a full saturated phenotypic model to the combined data of the multiple raters of twins' ADHD-like behaviour. The maximum number of parameters for the saturated model is 220 in total. A phenotypic saturated model-Cholesky decomposition-has (for 8 variables: 4 per twin) a lower matrix of $8 \times 8$, which has 36 variance/ covariance matrices per group (5: MZ males; MZ females; DZ males; DZ females; and DZ opposite sex pairs) and 8 means per group. After fitting the saturated model, we then fitted the Cholesky and both common pathway models. ADE models previously fitted to data on hyperactiveimpulsive and inattentive ratings on this sample indicated that we do not have the power to distinguish between $\mathrm{A}$ and D (Wood et al. 2009), as the sample size is relatively small for a twin analysis and therefore we have modelled only the $\mathrm{A}, \mathrm{C}$ and $\mathrm{E}$ influences on the behaviour.

\section{Results}

Principal component analysis previously conducted on these data indicated that the individual items map onto the hyperactivity-impulsivity and inattention subscales (Wood et al. 2009). Table 1 presents the means, standard deviations and ranges (prior to transformation) of each dimension for each sex and zygosity group, as rated by parents and teachers. Two (sex) by two (zygosity) analyses of variance (ANOVAs) were conducted on parent and teacher ratings. These analyses showed that boys scored significantly higher than girls on both the hyperactivity-impulsivity and inattention subscales for parent $[\mathrm{H}-\mathrm{I}$ : $\mathrm{F}(1$, $715)=29.70, p<0.0001$; Inattention: $\mathrm{F}(1,715)=21.56$, $p<0.0001]$ and teacher ratings $[\mathrm{H}-\mathrm{I}: \mathrm{F}(1,715)=48.52$, $p<0.0001$; Inattention: $\mathrm{F}(1,715)=52.72, p<0.001]$. For zygosity, a trend emerged for a difference in hyperactivity-impulsivity $[\mathrm{F}(1,715)=3.24, p=0.07]$ for teacher ratings, with MZs tending to score higher than DZs, but this was not observed for hyperactivity-impulsivity as rated by parents $[\mathrm{F}(1,715)=0.003, p=0.96]$ or inattention for either parent $[\mathrm{F}(1,715)=1.27, p=0.26]$ or 
A

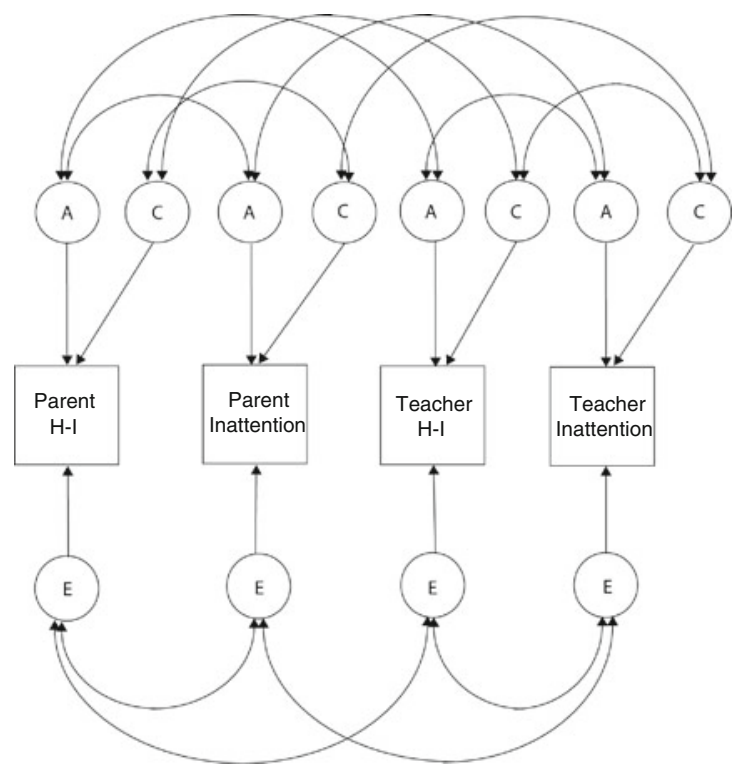

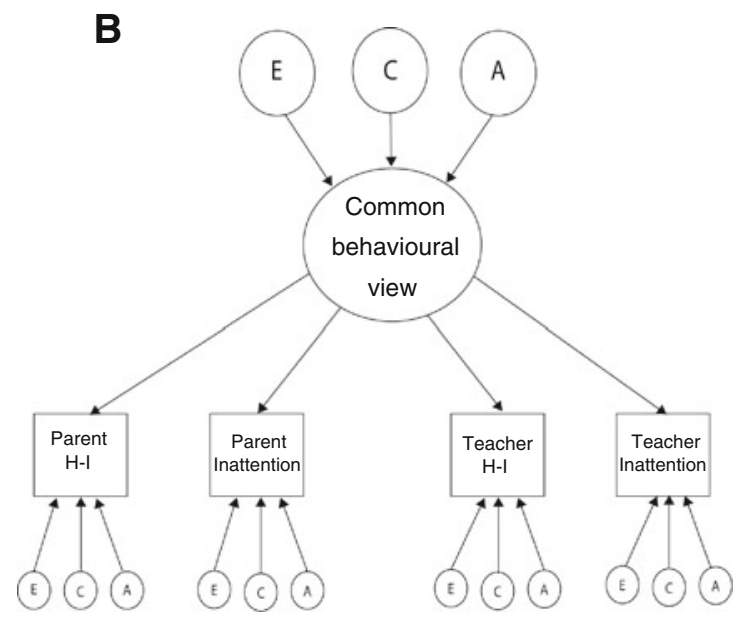

Fig. 1 Correlated factors/biometric model, presented in the form of a correlated factors model (a); common pathway/psychometric model with one latent factor (b); common pathway/psychometric model with two latent factors (c), all illustrated for one twin per pair. $A$ additive

teacher ratings $[\mathrm{F}(1,715)=2.11, p=0.15]$. A significant sex by zygosity interaction emerged for hyperactivityimpulsivity as rated by teachers $[\mathrm{F}(1,715)=4.75, p=0$. $03]$ but not for inattention $[\mathrm{F}(1,715)=0.04, p=0.85]$. No sex by zygosity interaction emerged for either dimension as rated by parents $[\mathrm{H}-\mathrm{I}: \mathrm{F}(1,715)=1.60, p<0.21$; Inattention: $\mathrm{F}(1,715)=1.99, p<0.16]$.

\section{Multi-rater models}

Cross-rater and cross-trait phenotypic correlations are shown in Table 2 and the cross-trait cross-twin (CTCT) correlations are shown in Table 3. The MZ CTCT correlations (Twin 1's hyperactive-impulsive score correlated with

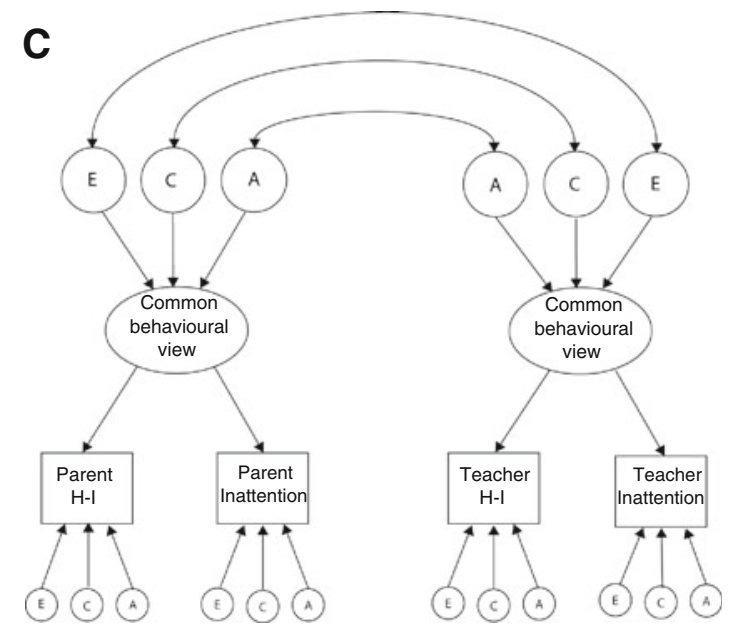

genetic influences, $C$ shared environmental influences, $E$ nonshared environmental influences. Parent refers to the primary caregiver. $\mathrm{H}-\mathrm{I}$ hyperactivity-impulsivity

Twin 2's inattentive score, and Twin 2's hyperactiveimpulsive score correlated with Twin 1's inattentive score; Table 3) are higher than DZ CTCT correlations, indicating genetic influence on the phenotypic relationship between hyperactive-impulsive and inattentive behaviours for both parent and teacher ratings. The CTCT correlations for teacher ratings indicate a possible small effect of the shared environment on the phenotypic correlation, as the DZ CTCT correlations are somewhat greater than half the MZ CTCT correlations. The CTCT correlations for parent ratings suggest possible non-additive genetic or contrast effects, whereby parents tend to exaggerate differences between DZ twins, which often leads to low dizygotic twin correlations in parent-rated ADHD behaviours (Eaves et al. 2000; Simonoff 
Table 1 Means, standard deviations and ranges for the Conners' ADHD subscales by sex and zygosity

\begin{tabular}{llll}
\hline Twin 1 & Twin 2 & Range \\
Mean (SD) & Mean (SD) & \\
\hline
\end{tabular}

Parent

$\mathrm{MZ}$

Hyperactivity-impulsivity

$\begin{array}{llll}\text { Male } & 7.45(5.92) & 7.10(5.42) & 0-23 \\ \text { Female } & 5.28(4.38) & 4.60(3.93) & 0-20 \\ \begin{array}{l}\text { Inattention } \\ \text { Male }\end{array} & 6.80(5.78) & 6.93(5.22) & 0-26 \\ \text { Female } & 4.71(4.50) & 4.68(4.09) & 0-21 \\ \text { DZSS } & & & \end{array}$

Hyperactivity-impulsivity

Male $\quad 7.50(5.85)$

Female $\quad 4.76(4.27)$

Inattention

Male $\quad 6.58(5.51)$

Female $\quad 5.84(5.09)$

DZOS

Hyperactivity-impulsivity

\begin{tabular}{|c|c|c|c|}
\hline Male & $6.93(5.18)$ & & $0-24$ \\
\hline Female & & $4.99(4.78)$ & $0-25$ \\
\hline \multicolumn{4}{|c|}{ Inattention } \\
\hline Male & $7.56(6.01)$ & & $0-27$ \\
\hline Female & & $4.99(4.66)$ & $0-23$ \\
\hline \multicolumn{4}{|l|}{ eacher } \\
\hline \multicolumn{4}{|l|}{ MZ } \\
\hline \multicolumn{4}{|c|}{ Hyperactivity-impulsivity } \\
\hline Male & $3.65(4.70)$ & $3.59(4.89)$ & $0-25$ \\
\hline Female & $2.15(3.43)$ & $1.75(2.31)$ & $0-16$ \\
\hline \multicolumn{4}{|c|}{ Inattention } \\
\hline Male & $5.72(5.15)$ & $5.79(5.41)$ & $0-24$ \\
\hline Female & $3.31(3.79)$ & $3.32(3.62)$ & $0-21$ \\
\hline \multicolumn{4}{|l|}{ ZSS } \\
\hline \multicolumn{4}{|c|}{ Hyperactivity-impulsivity } \\
\hline Male & $5.54(6.24)$ & $3.90(5.09)$ & $0-25$ \\
\hline Female & $2.21(3.70)$ & $1.49(3.07)$ & $0-25$ \\
\hline \multicolumn{4}{|c|}{ Inattention } \\
\hline Male & $6.65(6.21)$ & $6.18(5.95)$ & $0-27$ \\
\hline Female & $3.58(4.03)$ & $3.72(4.14)$ & $0-24$ \\
\hline \multicolumn{4}{|l|}{ ZOS } \\
\hline \multicolumn{4}{|c|}{ Hyperactivity-impulsivity } \\
\hline Male & $4.40(5.70)$ & & $0-27$ \\
\hline Female & & $2.64(3.82)$ & $0-19$ \\
\hline \multicolumn{4}{|c|}{ Inattention } \\
\hline Male & $6.96(7.10)$ & & $0-27$ \\
\hline Female & & $3.95(5.05)$ & $0-25$ \\
\hline
\end{tabular}

Means and standard deviations prior to transformation

$M Z$ monozygotic, DZSS dizygotic same-sex twin pairs, DZOS dizygotic opposite-sex twin pairs et al. 1998; Rietveld et al. 2004). Our sample size, however, is not sufficient to test for contrast effects or to distinguish between A and D influences on the two dimensions of ADHD separately in these models (Wood et al. 2009). As a result the estimates of $\mathrm{A}$ from these analyses refer to both narrow and broad sense heritability, which includes the effects of both additive and dominant genetic effects. The common pathway model with two latent factors-one for hyperactivityimpulsivity and one for inattention-was the best fit when the AIC values were compared with the saturated model (Table 4). To attain the most parsimonious model, parameters that did not significantly contribute (overlap with 0 ) to the fit of the model were dropped-in this case, parameters for shared environmental factors common to both raters and those unique to each rater. The AE model is nested within the full ACE model (i.e., subsets of the AE parameters are contained in the full ACE model). Dropping the $\mathrm{C}$ parameters (both specific and shared) from the model (Fig. 2) improved the fit (Table 4).

\section{Parameter estimates}

When examining the standardised parameter estimates for the best-fitting model (Fig. 2), the proportion of the total variance in hyperactivity-impulsivity scores due to a shared behavioural view was significant for both parents $(48 \%)$ and teachers $(28 \%)$. In accordance with these estimates, the proportion of variance due to unique rater view was higher for teachers $(73 \%)$ than for parents $(50 \%)$. The parameter estimates suggest that while parents are observing somewhat similar hyperactive-impulsive behaviour in these children as teachers, to a larger extent teachers have a unique view of this behaviour. Similarly, for inattention, the proportion of the total phenotypic variance explained due to a shared behavioural view was $61 \%$ for parents and $33 \%$ for teachers, whereas the proportion due to unique rater view was $39 \%$ for parents and $67 \%$ for teachers. Again, the parameter estimates suggest that parents are observing somewhat similar inattentive behaviour to teachers, while teachers are to a greater extent observing unique inattentive behaviours. This finding is in accordance with the heritability estimates for the unique behavioural views: in particular the A estimate for parents' unique behavioural view of inattention is low (8\%), which suggests that most of the genetic influences are contributing to the trait variance shared with teachers in the common behavioural view.

Examination of the $\mathrm{A}$ and $\mathrm{E}$ estimates for the shared behavioural view between parents and teachers, indicates a significantly higher genetic influence on hyperactivityimpulsivity (0.87) than inattentiveness (0.62) since the confidence intervals for these estimates do not overlap (Fig. 2). In terms of the genetic overlap between 
Table 2 Pearson correlations: males below, females above, diagonal

\begin{tabular}{|c|c|c|c|c|}
\hline & $\begin{array}{l}\text { Parent hyperactivity- } \\
\text { impulsivity }\end{array}$ & $\begin{array}{l}\text { Parent } \\
\text { inattention }\end{array}$ & $\begin{array}{l}\text { Teacher hyperactivity- } \\
\text { impulsivity }\end{array}$ & $\begin{array}{l}\text { Teacher } \\
\text { inattention }\end{array}$ \\
\hline Parent hyperactivity-impulsivity & & 0.48 & 0.37 & 0.13 \\
\hline Parent inattention & 0.65 & & 0.18 & 0.33 \\
\hline $\begin{array}{l}\text { Teacher hyperactivity- } \\
\text { impulsivity }\end{array}$ & 0.40 & 0.34 & & 0.44 \\
\hline Teacher inattention & 0.26 & 0.42 & 0.57 & \\
\hline
\end{tabular}

Table 3 Univariate, phenotypic and cross-trait cross-twin correlations on hyperactivity-impulsivity and inattention

\begin{tabular}{llr}
\hline & Hyperactivity-impulsivity & Inattention \\
\hline Parent & & \\
MZ & $0.76(0.70$ to 0.81$)$ & $0.52(0.42$ to 0.61$)$ \\
$\quad$ Male & $0.75(0.69$ to 0.80$)$ & $0.59(0.51$ to 0.67$)$ \\
Female & & \\
DZ & $0.22(0.10$ to 0.38$)$ & $0.03(-0.11$ to 0.17$)$ \\
Male & $0.24(0.10$ to 0.38$)$ & $0.02(-0.13$ to 0.16$)$ \\
Female & $0.14(0.01$ to 0.27$)$ & $0.04(-0.10$ to 0.17$)$ \\
DZ opposite-sex &
\end{tabular}

\begin{tabular}{|c|c|c|}
\hline & Cross-trait phenotypic correlations & Cross-trait cross-twin correlations \\
\hline \multicolumn{3}{|l|}{ MZ } \\
\hline Male & $0.60(0.52$ to 0.68$)$ & $0.46(0.36$ to 0.56$)$ \\
\hline Female & $0.58(0.50$ to 0.66$)$ & $0.46(0.36$ to 0.56$)$ \\
\hline \multicolumn{3}{|l|}{ DZ } \\
\hline Male & $0.66(0.58$ to 0.74$)$ & 0.15 (0.01 to 0.29$)$ \\
\hline Female & $0.50(0.39$ to 0.61$)$ & $0.13(-0.02$ to 0.27$)$ \\
\hline \multirow[t]{2}{*}{ DZ opposite-sex } & $0.58(0.48$ to 0.66$)$ & $0.26(0.13$ to 0.39$)$ \\
\hline & Hyperactivity-impulsivity & Inattention \\
\hline \multicolumn{3}{|l|}{ Teacher } \\
\hline \multicolumn{3}{|l|}{ MZ } \\
\hline Male & $0.53(0.43$ to 0.62$)$ & $0.48(0.37$ to 0.58$)$ \\
\hline Female & $0.60(0.51$ to 0.68$)$ & $0.68(0.60$ to 0.74$)$ \\
\hline \multicolumn{3}{|l|}{ DZ } \\
\hline Male & $0.39(0.25$ to 0.52$)$ & $0.13(-0.02$ to 0.28$)$ \\
\hline Female & $0.34(0.19$ to 0.48$)$ & $0.23(0.07$ to 0.38$)$ \\
\hline DZ opposite-sex & $0.40(0.27$ to 0.52$)$ & $0.27(0.12$ to 0.40$)$ \\
\hline
\end{tabular}

\begin{tabular}{lll}
\hline & Cross-trait phenotypic correlations & Cross-trait cross-twin correlations \\
\hline MZ & & $0.28(0.15$ to 0.40$)$ \\
$\quad$ Male & $0.57(0.47$ to 0.65$)$ & $0.23(0.10$ to 0.36$)$ \\
Female & $0.36(0.25$ to 0.47$)$ & $0.20(0.05$ to 0.35$)$ \\
DZ & & $0.13(-0.04$ to 0.28$)$ \\
Male & $0.61(0.50$ to 0.69$)$ & $0.24(0.10$ to 0.40$)$ \\
Female & $0.45(0.31$ to 0.57$)$ & \\
DZ opposite-sex & $0.59(0.49$ to 0.67$)$ & \\
\hline
\end{tabular}

Phenotypic correlation, within-twin correlation of hyperactivity-impulsivity and inattentiveness; cross-trait cross-twin correlation, hyperactivityimpulsivity in Twin 1 correlated with inattentiveness in Twin 2 
Table 4 Multivariate model-fitting results on the Conners' subscales of hyperactivity-impulsivity and inattention as rated by parents and teachers

\begin{tabular}{|c|c|c|c|c|c|c|c|c|}
\hline Model type & Details & $-2 \mathrm{LL}$ & df & Par & $\chi^{2}(\Delta \mathrm{df})$ & AIC & $p$ value & Comparison model \\
\hline \multirow[t]{7}{*}{ Saturated } & & 22284.10 & 4676 & 220 & & & & \\
\hline & CF (biometric) & 23226.20 & 4854 & 42 & $942.10(178)$ & 600.10 & $<0.0001$ & Saturated \\
\hline & $\begin{array}{l}\mathrm{CP} \text { (psychometric): } \\
\text { single latent factor }\end{array}$ & 22952.52 & 4866 & 31 & $668.42(190)$ & 288.42 & $<0.0001$ & Saturated \\
\hline & $\begin{array}{l}\mathrm{CP} \text { (psychometric): } \\
\text { two latent factors }\end{array}$ & 22943.00 & 4863 & 37 & $658.90(187)$ & 284.90 & $<0.0001$ & Saturated \\
\hline & $\begin{array}{l}\mathrm{CP} \text { (psychometric): } \\
\text { two latent factors } \\
\text { (no specific } \mathrm{C})\end{array}$ & 22946.54 & 4867 & 33 & $\begin{array}{l}662.44 \\
\quad(191) / 3.54(4)\end{array}$ & $280.44 /-4.46$ & $<0.0001 / 0.50$ & $\begin{array}{l}\text { Saturated/CP } \\
\text { (psychometric): } \\
\text { two latent factors }\end{array}$ \\
\hline & $\begin{array}{l}\mathrm{CP} \text { (psychometric) model: } \\
\text { two latent factors } \\
\text { (no shared } \mathrm{C})\end{array}$ & 22943.00 & 4866 & 34 & $\begin{array}{l}658.90 \\
(190) /-(4)\end{array}$ & $278.90 /-4.00$ & $<0.0001 / 1.0$ & $\begin{array}{l}\text { Saturated/CP } \\
\quad \text { (psychometric): } \\
\text { two latent factors }\end{array}$ \\
\hline & $\begin{array}{l}\mathrm{CP} \text { (psychometric) model: } \\
\text { two latent factors } \\
\text { (no C-specific or } \\
\text { shared) }\end{array}$ & 22946.54 & 4870 & 30 & $\begin{array}{l}662.44 \\
\quad(194) / 3.54(7)\end{array}$ & $274.44 /-3.46$ & $<0.0001 / 0.80$ & $\begin{array}{l}\text { Saturated/CP } \\
\text { (psychometric): } \\
\text { two latent factors }\end{array}$ \\
\hline
\end{tabular}

$C F$ correlated factors model, $C P$ common pathway model, $-2 L L \log$ likelihood fit statistic, $d f$ degrees of freedom, Par number of parameters, $\chi^{2}$ with $\Delta d f$ comparing model to the saturated model; AIC Akaike information criterion

${ }^{a}$ Best fitting model

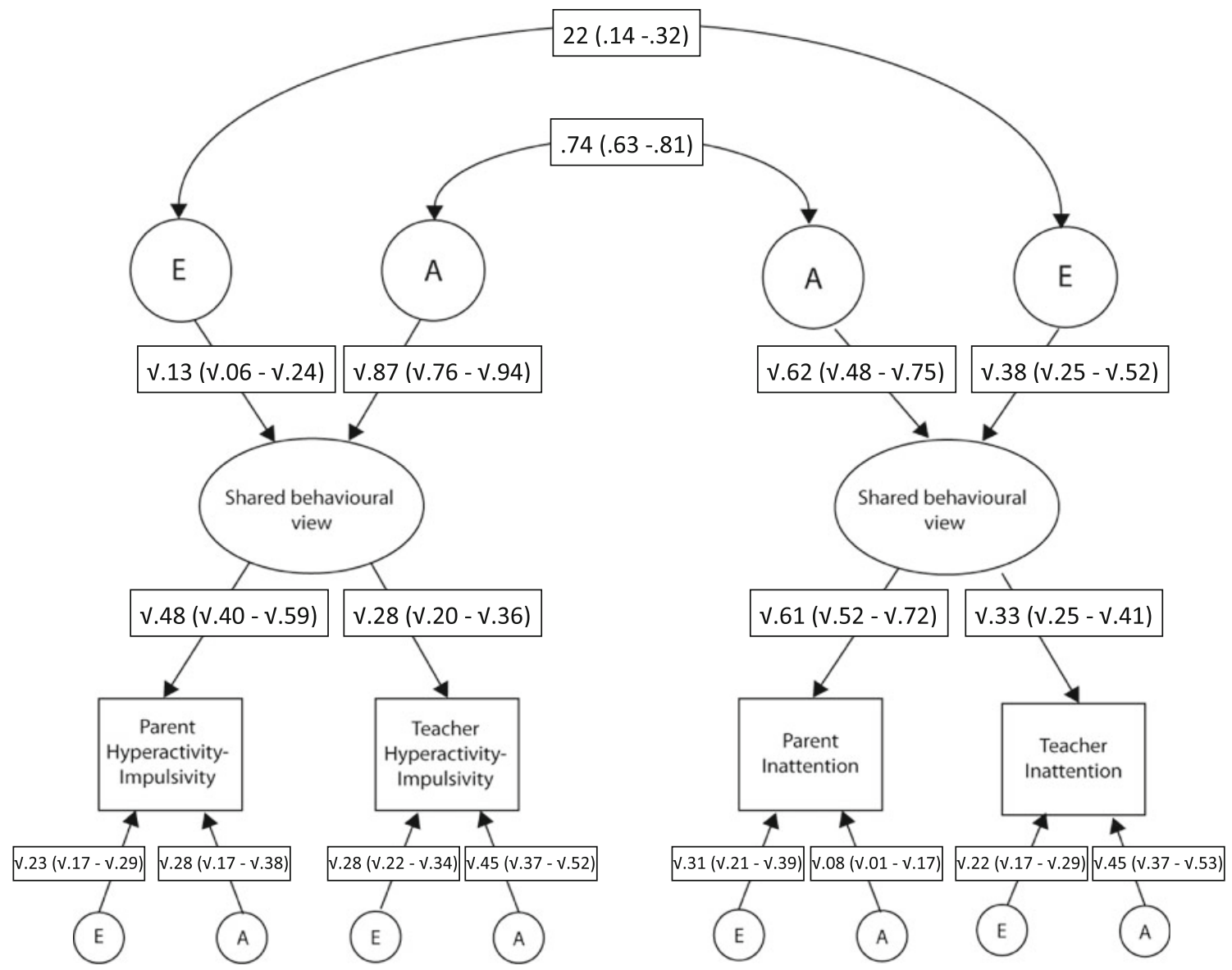

Fig. 2 Parameter estimates for common pathway model for parent and teacher reports of hyperactivity-impulsivity and inattention. Estimates of genetic correlations (rG) and nonshared environmental correlations (rE) are represented by double-headed arrows. Parent refers to the primary caregiver 
hyperactivity-impulsivity and inattention as rated by parents and teachers combined (the shared behavioural view), the genetic correlation was 0.74 (Fig. 2). The phenotypic correlation between the two behavioural domains emerged as 0.59 and the contribution of genetic influences to this correlation (rPh due to A) was 0.54; whereas the contribution of non-shared environmental influence $(\mathrm{rPh}$ due to E) was 0.05 . It was not possible to obtain the confidence intervals for these estimates due to restrictions in $\mathrm{Mx}$, but as the standardised parameter estimates which are used in the computation of the $\mathrm{rPh}, \mathrm{rPh}$-due-to- $\mathrm{A}$ and the $\mathrm{rPh}$-dueto-E are all significant (the CIs do not overlap with zero), the phenotypic correlation and the contribution of $\mathrm{A}$ and $\mathrm{E}$ to the phenotypic correlation must be significant. The standardised parameter estimates used in the computation of the $\mathrm{rPh}, \mathrm{rPh}$-due-to-A and the $\mathrm{rPh}$-due-to-E here are the genetic correlation between hyperactivity-impulsivity and inattention and their respective individual heritabilities.

\section{Discussion}

The use of multi-rater model fitting of parent and teacher data to investigate the aetiological overlap between the two dimensions of ADHD indicates that hyperactivity-impulsivity and inattention load on to two separate latent factors that are common to parents and teachers. These latent factors represent a common view of these behaviours between parents and teachers. Parents and teachers contribute to these common behavioural views of hyperactivity-impulsivity and inattention to varying extents. The model also included rater-specific factors, representing the unique view of parents and teachers. The loadings onto these rater specific factors indicate that, in addition to their shared perspective, parents and teachers report on different aspects of inattention and hyperactivity-impulsivity that are unique to the perspective of the two types of rater.

When parent and teacher ratings are combined to reflect a common view of children's ADHD symptoms, we found a substantial phenotypic overlap between the two symptom groups ( $\mathrm{rPh}=0.59)$; and that most of the genetic influences on hyperactivity-impulsivity were shared with inattention ( $\mathrm{rG}=0.74)$ (Neale and Maes 1996). The genetic correlation in this study is slightly higher than that reported in earlier studies that used parent ratings alone (McLoughlin et al. 2007) or looked at parent and teacher rating separately (Sherman et al. 1997). The higher estimate is likely to be due to the loading of parent and teacher ratings onto a shared view of these behaviours, which generates a more stable and consistent measure of inattention and hyperactivity-impulsivity in children. Although our results confirm the previous findings that the genetic influences on the two behavioural domains are largely shared, as in previous studies, they also indicate the presence of important unique genetic influences (Sherman et al. 1997; Levy et al. 2001; Eaves et al. 2000; Larsson et al. 2006; McLoughlin et al. 2007). These findings therefore also indicate partially distinct aetiologies for hyperactivity-impulsivity and inattention. This is in accordance with previous findings that the two symptom domains show distinct patterns of overlap with co-occurring behaviours; for example, oppositional behaviour overlaps mainly with hyperactivity-impulsivity (Wood et al. 2009), while reading ability overlaps mainly with inattention (Paloyelis et al. 2010).

The findings also indicate that parents and teachers rate largely comparable behaviours when reporting on inattentive and hyperactive-impulsive symptoms, but with additional unique behavioural views. For teacher ratings, the majority of the variance of both hyperactive-impulsive and inattentive behaviours was attributable to a unique view of these behaviours, whereas variance in parent ratings was more evenly split between that explained by a shared view with teachers and unique information. This finding suggests that teachers are observing, to a significant extent, different aspects of hyperactive-impulsive and inattentive behaviours in children. This is not surprising since teachers see children in the school environment, the behavioural demands of the classroom are quite different from the home environment, and children engage in activities at school that parents might not observe. Since these findings indicate some unique genetic effects on the parent and teacher ratings of child behaviour, etiological studies such as molecular genetic investigations could usefully focus on parent and teacher ratings separately, as well as the overlap between them.

The best fitting model indicates higher heritability estimates for ratings of hyperactivity-impulsivity (87\%) than inattention (62\%), which is in agreement with previous findings (Eaves et al. 1997; Goodman and Stevenson 1989; Kuntsi and Stevenson 2001). Since the 95\% confidence intervals for these heritability estimates do not overlap, this is likely to reflect a small but significant difference in the proportion of additive genetic influences on the two behavioural domains. The absence of rater specific shared environmental influences $(C)$ indicates an absence of rater bias and suggests that both parents and teachers are reliable reporters of behaviour when using DSM-IV symptom checklists (Saudino 2005).

The main strength of this study is the simultaneous analysis of teacher data and parent data in a multivariate model of inattention and hyperactivity-impulsivity in children. This study is an important addition to previous investigations since there was only one small earlier study of the two behavioural domains of ADHD using both parent and teacher ratings, and this is the first time the overlap between the domains has been examined using parent and teacher ratings simultaneously. The study is however restricted to ages 7-10 
so we are unable to comment on parent or teacher ratings at different developmental stages of ADHD. In addition, a larger sample would allow more complex analysis, such as separating out additive and dominance genetic effects and testing for contrast effects. As such, the heritability estimates in these models should be interpreted as including both narrow and broad sense heritability and future studies could test for both non-additive genetic effects and contrast effects. Furthermore, a larger sample would allow the investigation of sex-specific effects underlying the covariation in phenotypes (Neale and Kendler 1995). As with most other quantitative genetic studies of ADHD, the findings presented here relate to ADHD symptom scores that are continuously distributed throughout the population. Extrapolation of these results to clinical samples is based on the assumption that the ADHD diagnosis represents the impairing tail of the quantitative distributions of inattentive and hyperactive-impulsive symptoms; an assumption that is strongly supported by family and twin studies of ADHD (Chen et al. 2008). Future investigations of the similarities and differences in parent and teacher ratings could conduct latent class analysis on individual items of parent and teacher ratings, to identify whether parents and teachers are observing clusters of behavioural symptoms that fall into comparable groups.

In summary, this study is the first to utilise multi-rater twin modelling to examine the effects of different raters on the two behavioural dimensions of ADHD. Our results suggest that hyperactivity-impulsivity and inattention load onto separate latent factors that represent a common behavioural view for both parents and teachers. For both symptom groups, our findings indicated that teacher ratings may be better at identifying unique behaviours in both hyperactivity-impulsivity and inattention that parents cannot observe in the home. In addition, the results confirm that a large proportion of the genetic effects are shared between the symptom dimensions of ADHD, yet unique aetiological influences still remain. These findings indicate the value of studies that focus on both the overlap between, and the unique effects on, the two symptom domains and further the value of looking for different cognitive processes that mediate both the overlap and distinction identified at the level of genetic risk factors. This may be best achieved using population twin studies that include cognitiveexperimental, behavioural and molecular genetic data.

\section{References}

Akaike H (1987) Factor analysis and AIC. Psychometrika 52:317-332 American Psychiatric Association (2000) Diagnostic and statistical manual of mental disorders, 4th edn, text revised. American Psychiatric Association, Washington

Bartels M, Boomsma DI, Hudziak JJ, Rietveld MJH, van Beijsterveldt TCEM, van den Oord EJCG (2004) Disentangling genetic, environmental, and rater effects on internalizing and externalizing problem behavior in 10-year-old twins. Twin Res 7: $162-175$

Burns GL, Walsh JA, Owen SM, Snell J (1997) Internal validity of attention deficit hyperactivity disorder, oppositional defiant disorder, and overt conduct disorder symptoms in young children: implications from teacher ratings for a dimensional approach to symptom validity. J Clin Child Psychol 26:266-275

Chen W, Zhou KX, Sham P, Franke B, Kuntsi J, Campbell D et al (2008) DSM-IV combined type ADHD shows familial association with sibling trait scores: a sampling strategy for QTL linkage. Am J Med Genet B Neuropsychiatr Genet 147B: $1450-1460$

Conners CK (1998) Rating scales in attention-deficit/hyperactivity disorder: use in assessment and treatment monitoring. J Clin Psychiatry 59(Suppl 7):24-30

Conners CK, Sitarenios G, Parker JD, Epstein JN (1998a) Revision and restandardization of the Conners Teacher Rating Scale (CTRS-R): factor structure, reliability, and criterion validity. J Abnorm Child Psychol 26:279-291

Conners CK, Sitarenios G, Parker JD, Epstein JN (1998b) The revised Conners' Parent Rating Scale (CPRS-R): factor structure, reliability, and criterion validity. J Abnorm Child Psychol 26: 257-268

Eaves LJ, Silberg JL, Meyer JM, Maes HH, Simonoff E, Pickles A et al (1997) Genetics and developmental psychopathology. 2. The main effects of genes and environment on behavioral problems in the Virginia Twin Study of Adolescent Behavioral Development. J Child Psychol Psychiatry 38:965-980

Eaves L, Rutter M, Silberg JL, Shillady L, Maes H, Pickles A (2000) Genetic and environmental causes of covariation in interview assessments of disruptive behavior in child and adolescent twins. Behav Genet 30:321-334

Goodman R, Stevenson J (1989) A twin study of hyperactivity. II. The aetiological role of genes, family relationships and perinatal adversity. J Child Psychol Psychiatry 30:691-709

Hartman CA, Rhee SH, Willcutt EG, Pennington BF (2007) Modeling rater disagreement for ADHD: are parents or teachers biased? J Abnorm Child Psychol 35(4):536-542

Hewitt JK, Silberg JL, Neale MC, Eaves LJ, Erickson M (1992) The analysis of parental ratings of children's behavior using LISREL. Behav Genet 22:293-317

Holmes J, Payton A, Barrett J, Harrington R, McGuffin P, Owen M et al (2002) Association of DRD4 in children with ADHD and comorbid conduct problems. Am J Med Genet 114:150-153

Hudziak JJ, Heath AC, Madden PF, Reich W, Bucholz KK, Slutske W et al (1998) Latent class and factor analysis of DSM-IV ADHD: a twin study of female adolescents. J Am Acad Child Adolesc Psychiatry 37:848-857

Kuntsi J, Asherson P (2005) An interdisciplinary approach to ADHD. In: Larimer MP (ed) Attention deficit hyperactivity disorder research developments. Nova Science, Hauppauge, NY, pp 1-30

Kuntsi J, Stevenson J (2001) Psychological mechanisms in hyperactivity. II. The role of genetic factors. J Child Psychol Psychiatry 42:211-219

Kuntsi J, Rogers H, Swinard G, Borger N, van der Meere J, Rijsdijk F et al (2006) Reaction time, inhibition, working memory and 'delay aversion' performance: genetic influences and their interpretation. Psychol Med 36:1613-1624

Larsson H, Lichtenstein P, Larsson JO (2006) Genetic contributions to the development of ADHD subtypes from childhood to adolescence. J Am Acad Child Adolesc Psychiatry 45:973-981

Levy F, McStephen M, Hay DA (2001) The diagnostic genetics of ADHD symptoms and subtypes. In: Levy F, Hay D (eds) Attention, genes and ADHD. Taylor \& Francis, Philadelphia, pp 35-58 
Martin N, Scourfield J, McGuffin P (2002) Observer effects and heritability of childhood attention-deficit hyperactivity disorder symptoms. Br J Psychiatry 180:260-265

McLoughlin G, Ronald A, Kuntsi J, Asherson P, Plomin R (2007) Genetic support for the dual nature of attention deficit hyperactivity disorder: substantial genetic overlap between the inattentive and hyperactive-impulsive components. J Abnorm Child Psychol 35:999-1008

Nadder TS, Silberg JL, Rutter M, Maes HH, Eaves LJ (2001) Comparison of multiple measures of ADHD symptomatology: a multivariate genetic analysis. J Child Psychol Psychiatry 42: $475-486$

Neale MC (1997) Mx: statistical modelling [computer software]. Department of Psychiatry, Medical College of Virginia, Richmond

Neale MC, Cardon LR (1992) Methodology for genetic studies of twins and families. Kluwer, Dordrecht

Neale MC, Kendler KS (1995) Models of comorbidity for multifactorial disorders. Am J Hum Genet 57:935-953

Neale MC, Maes HH (1996) Methodology for genetics studies of twins and families, 6th edn. Kluwer, Dordrecht

Neale MC, Miller MB (1997) The use of likelihood-based confidence intervals in genetic models. Behav Genet 27:113-120

Oliver B, Plomin R (2006) Twins' Early Development Study (TEDS): a multivariate, longitudinal genetic investigation of language, cognition and behavior problems from childhood through adolescence. Twin Res Hum Genet 10(1):96-105

Paloyelis Y, Rijsdijk F, Wood AC, Asherson P, Kuntsi J (2010) The genetic association between ADHD symptoms and reading difficulties: the role of inattentiveness and IQ. J Abnorm Child Psychol 38(8):1083-1095

Pelham WE Jr, Gnagy EM, Greenslade KE, Milich R (1992) Teacher ratings of DSM-III-R symptoms for the disruptive behavior disorders. J Am Acad Child Adolesc Psychiatry 31:210-218

Plomin R, DeFries JC, McClearn GE, McGuffin P (2001) Behavioural genetics. Worth, New York

Price TS, Freeman B, Craig I, Petrill SA, Ebersole L, Plomin R (2000) Infant zygosity can be assigned by parental report questionnaire data. Twin Res 3:129-133
Rietveld MJ, Hudziak JJ, Bartels M, van Beijsterveldt CE, Boomsma DI (2004) Heritability of attention problems in children: longitudinal results from a study of twins, age 3 to 12. J Child Psychol Psychiatry 45:577-588

Saudino KJ (2005) Rater bias models. In: Everitt B, Howell D (eds) Encyclopedia of statistics in behavioral science, vol 4. Wiley, Chichester, pp 1716-1717

Saudino KJ, Ronald A, Plomin R (2005) The etiology of behavior problems in 7-year-old twins: substantial genetic influence and negligible shared environmental influence for parent ratings and ratings by same and different teachers. J Abnorm Child Psychol 33:113-130

Sherman DK, Iacono WG, McGue MK (1997) Attention-deficit hyperactivity disorder dimensions: a twin study of inattention and impulsivity-hyperactivity. J Am Acad Child Adolesc Psychiatry 36:745-753

Simonoff E, Pickles A, Hervas A, Silberg JL, Rutter M, Eaves L (1998) Genetic influences on childhood hyperactivity: contrast effects imply parental rating bias, not sibling interaction. Psychol Med 28:825-837

Stata Corporation (1997) Stata statistical software (version release 9.0) [computer software]. Stata Corporation, College Station

Thapar A, Harrington R, Ross K, McGuffin P (2000) Does the definition of ADHD affect heritability? J Am Acad Child Adolesc Psychiatry 39:1528-1536

Trouton A, Spinath FM, Plomin R (2002) Twins early development study (TEDS): a multivariate, longitudinal genetic investigation of language, cognition and behavior problems in childhood. Twin Res 5:444-448

Van der Valk JC, Van Den Oord EJCG, Verhulst FC, Boomsma DI (2001) Etiology of 3-year-old twins' problem behaviors: different views or rater bias? J Child Psychol Psychiatry 42:921-931

Wood AC, Rijsdijk F, Asherson P, Kunst J (2009) Hyperactiveimpulsive symptom scores and oppositional behaviours reflect alternate manifestations of a single liability. Behav Genet 39: $447-460$ 